\title{
Dynamics of the Chaplygin Ball with Variable Parameters
}

\author{
A. V. Borisov, E. A. Mikishanina
}

This work is devoted to the study of the dynamics of the Chaplygin ball with variable moments of inertia, which occur due to the motion of pairs of internal material points, and internal rotors. The components of the inertia tensor and the gyrostatic momentum are periodic functions. In general, the problem is nonintegrable. In a special case, the relationship of the problem under consideration with the Liouville problem with changing parameters is shown. The case of the Chaplygin ball moving from rest is considered separately. Poincaré maps are constructed, strange attractors are found, and the stages of the origin of strange attractors are shown. Also, the trajectories of contact points are constructed to confirm the chaotic dynamics of the ball. A chart of dynamical regimes is constructed in a separate case for analyzing the nature of strange attractors.

Keywords: Chaplygin ball, Poincaré map, strange attractor, chart of dynamical regimes

Received July 22, 2020

Accepted August 20, 2020

The work was supported by RFBR grant 18-29-10051 mk and was carried out at MIPT under project 5-100 for state support for leading universities of the Russian Federation.

Alexei V. Borisov

borisov@rcd.ru

Moscow Institute of Physics and Technology (National Research University)

Institutskiy per. 9, Dolgoprudny, Moscow Region, 141701 Russia

Evgeniya A. Mikishanina

evaeva_84@mail.ru

Chuvash State University

Moskovskii prosp. 15, Cheboksary, 428015 Russia 


\section{Introduction}

In the classic version, the Chaplygin ball is understood as a dynamically asymmetric balanced rigid body with a spherical shape that rolls along a rough surface without slipping. Sergei Chaplygin was the first to conduct in 1903 a study of the dynamics of motion of such a ball [1]. A sufficient number of papers have been devoted to various generalizations of the Chaplygin ball problem [2-8]. The analysis of the trajectory of the point of contact of the ball with the surface on which it rolls is presented in the paper [9]. These studies are particularly relevant in robotics in relation to mobile spherical robotic devices [10-12].

This work is devoted to modeling the dynamics of the Chaplygin ball rolling without slipping with changing parameters. There are two nonholonomic models of ball rolling on a plane:

- the model of rolling without slipping (classical rolling model);

- the model of rolling without slipping and spinning (rubber rolling model).

In contrast, for example, to the works $[7,8]$, in which the rolling model without slipping and spinning (rubber rolling model) was studied in detail, this paper considers the classical Chaplygin ball model with variable moments of inertia. Usually, rotors or sliders are used to model the Chaplygin ball with variable parameters. Using the latter allows one to organize the control of the ball due to the variable gyrostatic momentum $[5,13]$. But in this work, we rely on the Liouville approach $[14,15]$, when moving solids located in the body cavity are replaced by material points moving according to a certain law. Thus, in this paper we are talking about a nonholonomic analog of the Liouville problem. This approach is more general and allows the problem to be explored in a general setting.

In this paper, we consider a dynamically asymmetric ball rolling without slipping, in which rotors rotate and pairs of internal material points move, so that the center of mass always coincides with the geometric center of the shell. Changing the moments of inertia of the body due to the motion of masses entails various effects [16]: the appearance of attracting sets and the appearance of strange attractors. Computer analysis of the resulting system dynamics and contact point trajectories is performed. The appearance of strange attractors is also shown. To analyze the nature of the appearance of strange attractors, a chart of dynamical regimes is constructed and the areas of transition to chaos are determined.

\section{The main equations}

\subsection{Equations of motion and first integrals}

Let's consider the problem of rolling along a horizontal plane of a dynamically asymmetric rigid body that has the shape of a ball and contains movable material points and rotors inside it (Fig. 1). For the body in question, we will make the following assumptions:

- the body rolls without slipping, the center of mass of the system is at the geometric center of the shell and the main axes of inertia of the system are stationary relative to the shell;

- the internal material points undergo periodic motions with the same period $\tau$, so that the center of mass and the main axes of inertia remain fixed relative to the shell;

- the rotors are axisymmetric and rotate with angular velocities that are $\tau$-periodic functions of time, and the axes of rotation of the rotors coincide with its symmetry axes, so that the rotation of the rotors does not influence the mass distribution of the system. 


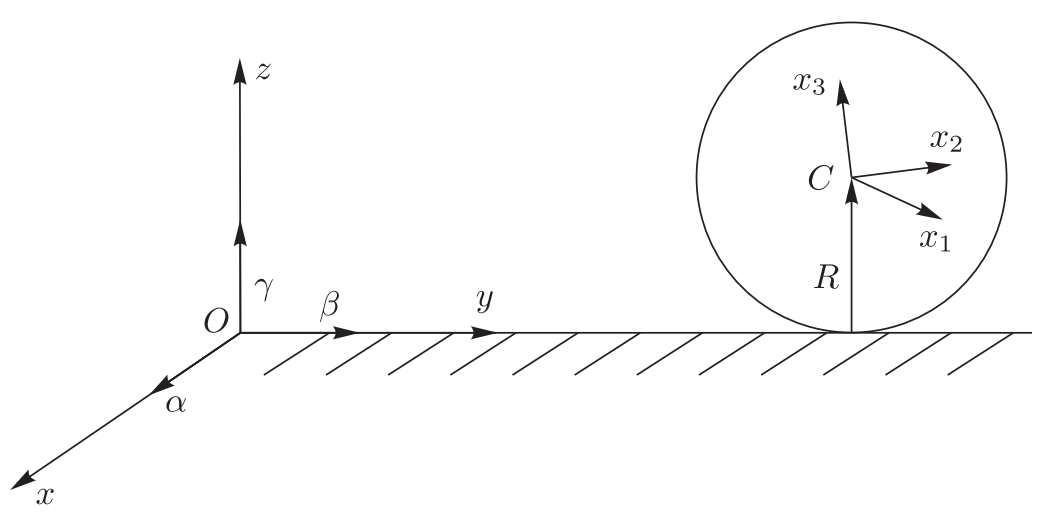

Fig. 1. Chaplygin ball.

We introduce two coordinate systems (Fig. 1):

- a fixed coordinate system $O x y z$ centered at a fixed point $O$, with the body moving in a plane orthogonal to the axis $O z$;

- a moving coordinate system $C x_{1} x_{2} x_{3}$ centered at point $C$, which is at the geometric center of the body, and the axes of this coordinate system are directed along the main geometric axes.

We denote the unit vectors of the coordinate axes of the fixed coordinate system $O x y z$ by $\boldsymbol{\alpha}, \boldsymbol{\beta}, \boldsymbol{\gamma}$, the translational and angular velocities of the spherical shell by $\boldsymbol{v}=\left(v_{1}, v_{2}, v_{3}\right)$ and $\boldsymbol{\omega}=\left(\omega_{1}, \omega_{2}, \omega_{3}\right)$, and the radius vector from the center of the ball to the point of contact $P$ by $R$, where $R=-a \gamma$ and $a$ is the radius of the ball.

The condition for no slippage at the point of contact has the form

$$
\boldsymbol{v}+\boldsymbol{\omega} \times \boldsymbol{R}=0 .
$$

The evolution of variables $\boldsymbol{\alpha}, \boldsymbol{\beta}, \boldsymbol{\gamma}$ follows from the condition of no slippage and is described by Poisson equations:

$$
\dot{\alpha}=\alpha \times \omega, \quad \dot{\beta}=\beta \times \omega, \quad \dot{\gamma}=\gamma \times \omega .
$$

The angular momentum of the ball relative to the point of contact $P$ has the form

$$
\boldsymbol{M}=\mathbf{I} \boldsymbol{\omega}+d \boldsymbol{\gamma} \times(\boldsymbol{\omega} \times \boldsymbol{\gamma})+\boldsymbol{k}, \quad d=m a^{2},
$$

where $\mathbf{I}=\mathbf{I}(t)$ is the central tensor of inertia of the entire system and $\boldsymbol{k}=\boldsymbol{k}(t)$ is the gyrostatic momentum vector resulting from the combined motion of the material points and the rotors.

For $d=0$ the equations will take the form similar to that of the equations in the Liouville problem, which is studied in $[14,15]$, including those with periodically changing components of the tensor of inertia and the gyrostatic momentum [15]. However, in the problem of the Chaplygin ball, such a case is practically impossible, since it corresponds to the motion of a ball of zero radius. Therefore, we will assume that $d \neq 0$.

The evolution of angular momentum is given by the equation

$$
\dot{M}=M \times \omega .
$$

Rewriting (2.2) in the form

$$
\boldsymbol{M}=(\mathbf{I}+d(\mathbf{E}-\boldsymbol{\gamma} \otimes \boldsymbol{\gamma})) \boldsymbol{\omega}+\boldsymbol{k},
$$


we get a closed system with respect to $\boldsymbol{\omega}, \boldsymbol{\gamma}$ :

$$
\begin{gathered}
\dot{\boldsymbol{M}}=\boldsymbol{M} \times \boldsymbol{\omega}, \quad \dot{\boldsymbol{\gamma}}=\boldsymbol{\gamma} \times \boldsymbol{\omega} \\
\boldsymbol{\omega}=\mathbf{A}(\boldsymbol{M}-\boldsymbol{k}), \quad \mathbf{A}=(\mathbf{I}+d(\mathbf{E}-\boldsymbol{\gamma} \otimes \gamma))^{-1} .
\end{gathered}
$$

The system (2.5) has three integrals of motion [18]

$$
\gamma^{2}=1, \quad M^{2}=G^{2}, \quad(M, \gamma)=H .
$$

Equations (2.1) and (2.5) form a complete system describing the dynamics of a Chaplygin ball rolling without slipping. The complete system, in addition to the above-mentioned first integrals, has two first integrals

$$
(\boldsymbol{M}, \boldsymbol{\alpha})=F, \quad(\boldsymbol{M}, \boldsymbol{\beta})=Q
$$

Let's consider a special case where the inertia tensor has constant components. If the gyrostatic momentum $\boldsymbol{k}=$ const, the system $(2.5)$ has additionally an energy integral $[2,18]$

$$
E=\frac{1}{2}(\boldsymbol{M}, \boldsymbol{\omega})
$$

and an invariant measure with density

$$
\mu(\boldsymbol{M}, \boldsymbol{\gamma})=\left(1-d\left(\boldsymbol{\gamma},(\mathbf{I}+d \mathbf{E})^{-1} \gamma\right)\right)^{-0.5}
$$

In this case, the system is integrable by quadratures according to the Euler - Jacobi theorem [17].

In the case where the components of the gyrostatic momentum $\boldsymbol{k}$ are constant values, the system (2.5) is also integrable (see [18] for more details) with an integrating factor (2.7).

Next, we will proceed to a detailed study of the case of the variable inertia tensor and the gyrostatic momentum. For simplicity, we assume that $(\boldsymbol{M}, \boldsymbol{\gamma})=0$.

\subsection{The point of contact}

The system of equations describing the position of the ball (the position of the contact point) on the plane $O x y$ has the form

$$
\begin{aligned}
\dot{\boldsymbol{\alpha}} & =\boldsymbol{\alpha} \times \boldsymbol{\omega}, & & \dot{\boldsymbol{\beta}}=\boldsymbol{\beta} \times \boldsymbol{\omega}, \\
\dot{X} & =a(\boldsymbol{\beta}, \boldsymbol{\omega}), & \dot{Y} & =-a(\boldsymbol{\alpha}, \boldsymbol{\omega}) .
\end{aligned}
$$

For convenience, we choose the unit vectors as follows:

$$
\alpha=\frac{M \times \gamma}{G}, \quad \beta=\frac{M}{G} .
$$

Then the equations for the trajectory of the contact point can be rewritten as

$$
\dot{X}=\frac{a}{G}(\boldsymbol{M}, \boldsymbol{\omega}), \quad \dot{Y}=\frac{a}{G}(\gamma \times M, \omega) .
$$

As the initial conditions for Eqs. (2.8), we take the values $X(0)=0, Y(0)=0$. 


\section{The case of the variable tensor of inertia and the gyrostatic momentum}

Due to the assumptions made about the system under consideration the inertia tensor has a diagonal form

$$
\mathbf{I}(t)=\operatorname{diag}\left(I_{1}(t), I_{2}(t), I_{3}(t)\right),
$$

and its components, as well as the components of the vector of gyrostatic momentum $\boldsymbol{k}(t)=$ $=\left(k_{1}(t), k_{2}(t), k_{3}(t)\right)$, are periodic functions with a period $\tau$ :

$$
\boldsymbol{k}(t+\tau)=\boldsymbol{k}(t), \quad \mathbf{I}(t+\tau)=\boldsymbol{I}(t) .
$$

Rewrite system (2.5) as

$$
\dot{M}=\boldsymbol{M} \times \mathbf{A}(\boldsymbol{M}-\boldsymbol{k}), \quad \dot{\gamma}=\boldsymbol{\gamma} \times \mathbf{A}(\boldsymbol{M}-\boldsymbol{k}) .
$$

If

$$
\mathbf{I}(-t)=\mathbf{I}(t), \quad \boldsymbol{k}(-t)=\boldsymbol{k}(t),
$$

then the system admits only the symmetry

$$
t \rightarrow t, \quad M \rightarrow M, \quad \gamma \rightarrow-\gamma
$$

If

$$
\mathbf{I}(-t)=\mathbf{I}(t), \quad \boldsymbol{k}(-t)=-\boldsymbol{k}(t),
$$

then the system allows symmetry

$$
t \rightarrow t, \quad M \rightarrow M, \quad \gamma \rightarrow-\gamma
$$

and two involutions

$$
\begin{array}{lll}
t \rightarrow-t, & M \rightarrow-M, & \gamma \rightarrow \gamma, \\
t \rightarrow-t, & M \rightarrow-M, & \gamma \rightarrow-\gamma .
\end{array}
$$

The presence of involutions in the system means that the system is reversible, which means that the attractors and repellers in the system arise in pairs and they are symmetric with respect to each of the involutions (3.3).

Next, assume that the two internal masses move symmetrically along the $C x_{2}$ axis, so that the tensor of inertia changes in accordance with the law

$$
\mathbf{I}(t)=\operatorname{diag}\left(I_{1}+(J+\delta \sin \Omega t)^{2}, I_{2}, I_{3}+(J+\delta \sin \Omega t)^{2}\right) .
$$

And the gyrostatic momentum vector has the form

$$
\boldsymbol{k}(t)=\left(k_{1} \sin \Omega t, k_{2} \sin \Omega t, k_{3} \sin \Omega t\right),
$$

where the vector $\left(k_{1}, k_{2}, k_{3}\right)$ is further denoted by $\boldsymbol{K}$.

Consider two cases. If at the initial instant of time $\boldsymbol{\omega}(0)=0$ and $\boldsymbol{k}(0)=0$, then we speak about the motion of the system from rest. Since the integral is preserved for system (2.5), the condition $M^{2}=G^{2}=0$ will be satisfied. Thus, we consider the case of the motion of the body in question from rest, i.e., $G=0$, as well as the general case. 


\subsection{Motion from rest. The Case $G=0$}

In the case of motion from rest, the system (2.5) with the tensor of inertia (3.4) and the gyrostatic momentum (3.5) will take the form

$$
\begin{aligned}
\dot{\boldsymbol{\gamma}} & =\boldsymbol{\gamma} \times(-\mathbf{A} \boldsymbol{k}(t)), \\
\mathbf{A} & =(\mathbf{I}+d(\mathbf{E}-\boldsymbol{\gamma} \otimes \boldsymbol{\gamma}))^{-1} .
\end{aligned}
$$

Only one integral of motion is preserved for system (3.5), and this system admits symmetry

$$
t \rightarrow t, \quad \gamma \rightarrow-\gamma
$$

And if the additional condition (3.2) is met, it also admits an involution

$$
t \rightarrow-t, \quad \gamma \rightarrow-\gamma .
$$

Numerically integrating the equations of motion (3.6) in variables, we construct Poincaré maps, recalculating the original variables when displaying the Poincaré map on the secant plane $t=0$ in the cylindrical coordinates $(z, l)$ :

$$
\gamma_{1}=\sqrt{1-z^{2}} \sin l, \quad \gamma_{2}=\sqrt{1-z^{2}} \cos l, \quad \gamma_{3}=z, \quad z \in[-1,1], \quad l \in[-\pi, \pi) .
$$

Numerical analysis of dynamics can be performed using a two-dimensional map for the period $\tau=\frac{2 \pi}{\Omega}$ in variables $(z, l)$.

In experiments for $J=0$ in the tensor of inertia, i.e., with the mass pair moving symmetrically relative to the center of mass, only periodic modes were detected.

When displayed, depending on the values of numeric parameters, the following may be present:

- fixed points corresponding to limit cycles in phase space;

- invariant curves corresponding to quasi-periodic solutions of the system (Fig. 2).

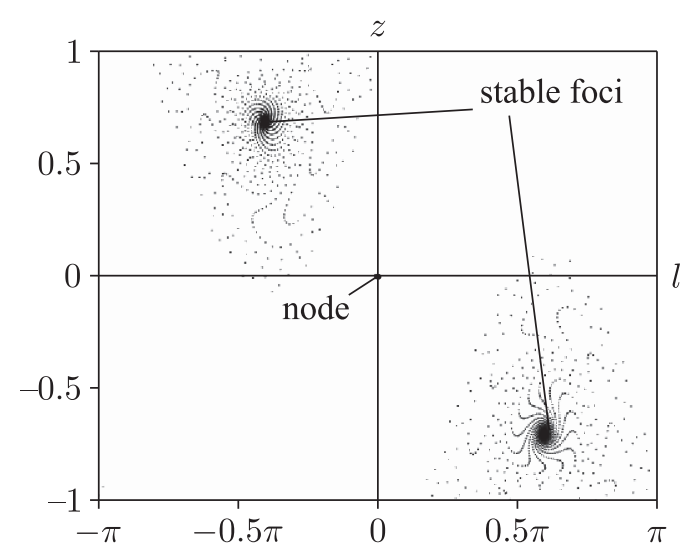

A

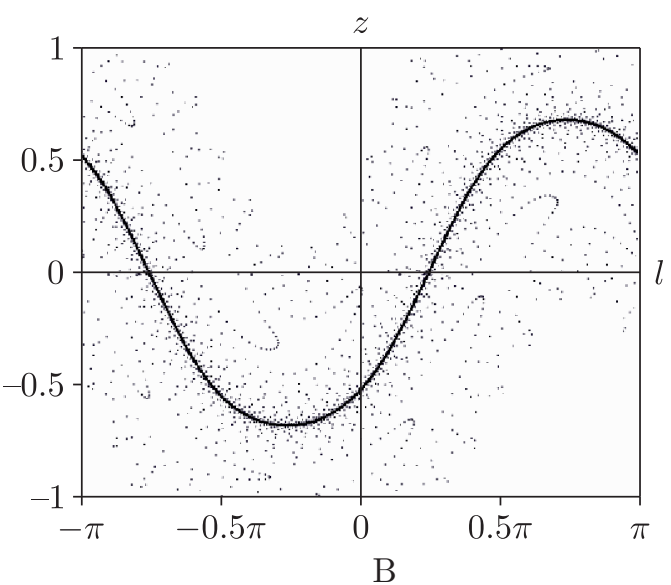

$\mathrm{B}$

Fig. 2. Poincaré maps for A: $\mathbf{I}=\operatorname{diag}(4,3,2), \boldsymbol{K}=(3,-1,-2), d=1.5, J=-0.5, \delta=1.2, \Omega=0.18$; B: $\mathbf{I}=\operatorname{diag}(4,3,2), \boldsymbol{K}=(3,-1,-2), d=1.5, J=-0.5, \delta=1.2, \Omega=0.4$.

In numerical experiments, no strange attractors were found, so the question of the presence of strange attractors in the Poincaré map in the case of a body rolling without slipping from rest remains open. 


\subsection{The Case $G \neq 0$}

As already mentioned above, the system (2.5) has in general only three integrals of motion and is nonintegrable by quadratures. We introduce the Andoyer-Deprit variables $(z, l, g)[19]$ :

$$
\begin{aligned}
& M_{1}=G \sqrt{1-z^{2}} \sin l, \quad M_{2}=G \sqrt{1-z^{2}} \cos l, \quad M_{3}=G z, \\
& \gamma_{1}=z \cos g \sin l+\sin g \cos l, \quad \gamma_{2}=z \cos g \cos l-\sin g \sin l, \quad \gamma_{3}=-\sqrt{1-z^{2}} \cos g,
\end{aligned}
$$

where $z \in[-1,1], l, g \in[-\pi, \pi)$.

For further constructions, we will set the inertia tensor as (3.4), where $J=0, I_{1}=4$, $I_{2}=3, I_{3}=2$. Thus, moving masses move symmetrically relative to the center of mass of the body. In the case where $\delta<1$, the tensor of inertia is not interesting for such a task, since the relation is preserved: $I_{1}(t)>I_{2}(t)>I_{3}(t)$. It is much more interesting to investigate the case where this ratio is periodically violated. So let's take $\delta=2$. Graphs of the components of the tensor of inertia are shown in Fig. 3.

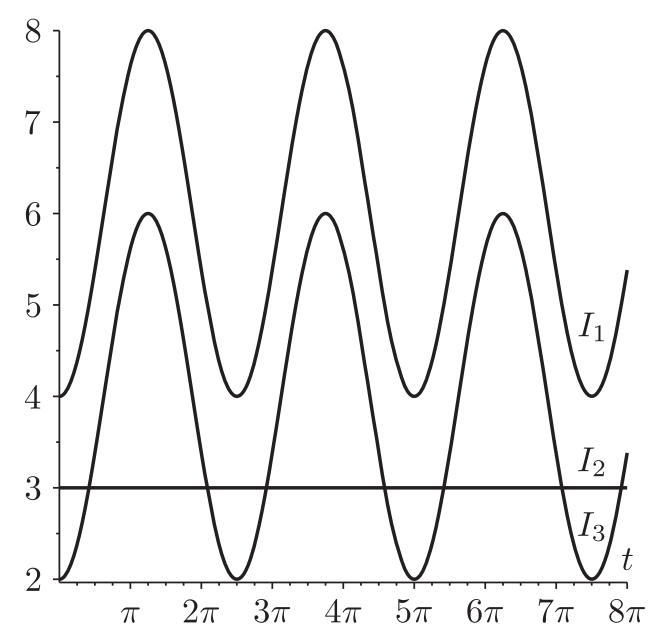

Fig. 3. Components of the tensor of inertia for $\mathbf{I}=\operatorname{diag}(4,3,2), J=0, \delta=2, \Omega=0.4$.

Since the equations of motion are nonautonomous, it is possible to construct a threedimensional map for the period $\tau=\frac{2 \pi}{\Omega}$ in variables $(z, l, g)$ with a secant plane $t=0$.

The system can make chaotic fluctuations for $G \neq 0$. In some cases, starting with chaotic fluctuations, the system comes to a quasi-periodic mode. Attractive invariant curves appear in the maps (Fig. 4).

In other cases, it is possible for dissipation to appear in the system, which leads to the appearance of strange attractors in the Poincaré maps.

Let's define Lyapunov exponents for the three-dimensional map (Fig. 5):

$$
\lambda_{1} \approx 0.041 \pm 0.009, \quad \lambda_{2} \approx-0.0033 \pm 0.0011, \quad \lambda_{3} \approx-0.075 \pm 0.006
$$

The magnitude of the indicators speaks in favor of a strange attractor.

The question of the appearance of the mechanism of chaotic attractors remains open. A doubling of period 7-14 was detected (Fig. 6) in the chart of dynamical regimes, however, due to the already large size of the periods, further doubling is quite difficult to track. Therefore, it is possible to make an assumption about the Feigenbaum mechanism of the appearance of the attractor [20]. 

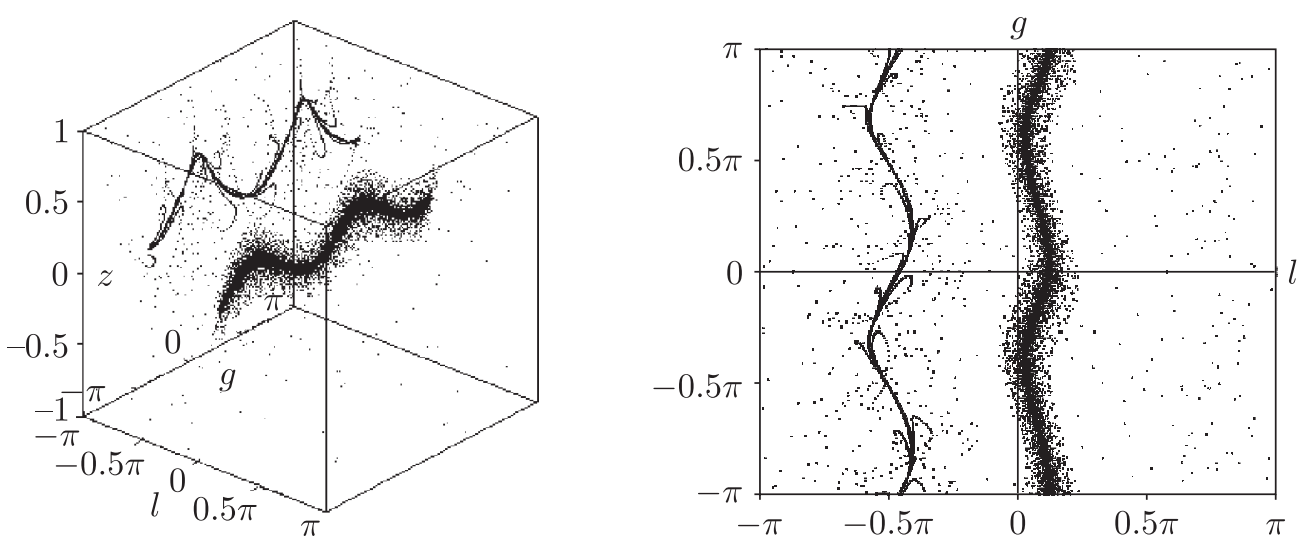

Fig. 4. The Poincaré map for $\mathbf{I}=\operatorname{diag}(4,3,2), \boldsymbol{K}=(-4,-5,3), d=4.5, J=0, \delta=2, \Omega=2, G=3$.
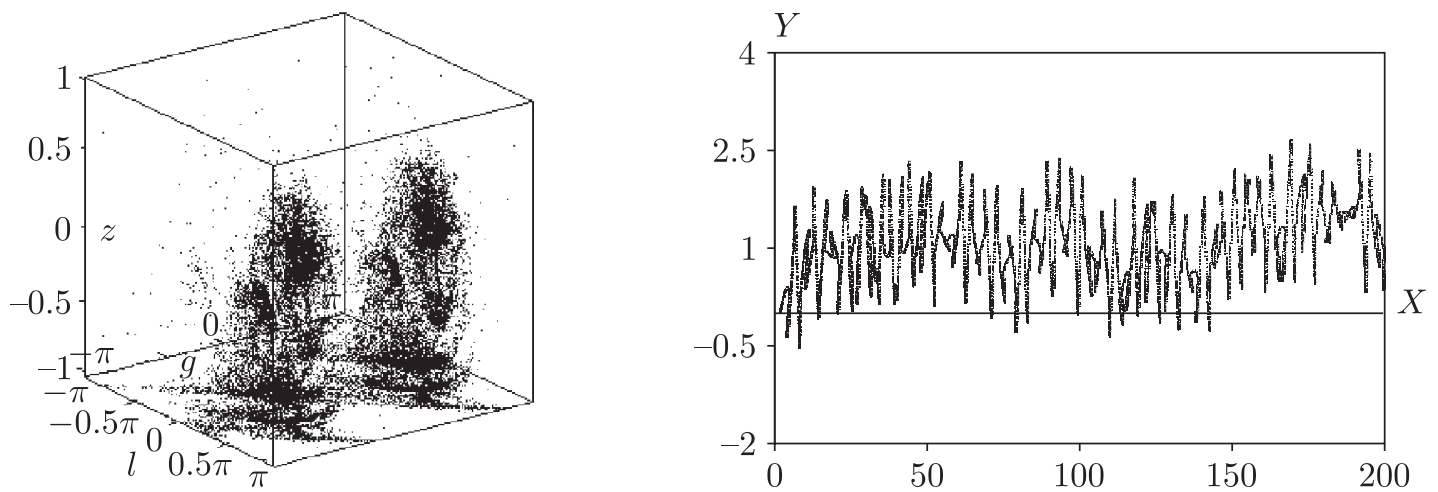

Fig. 5. The Poincaré map for $\mathbf{I}=\operatorname{diag}(4,3,2), \boldsymbol{K}=(-6,-3,-9), d=4.385, J=0, \delta=2, \Omega=1.07$, $G=3$ and the trajectory of the contact point for $g(0)=1, l(0)=1, z(0)=0.5$.

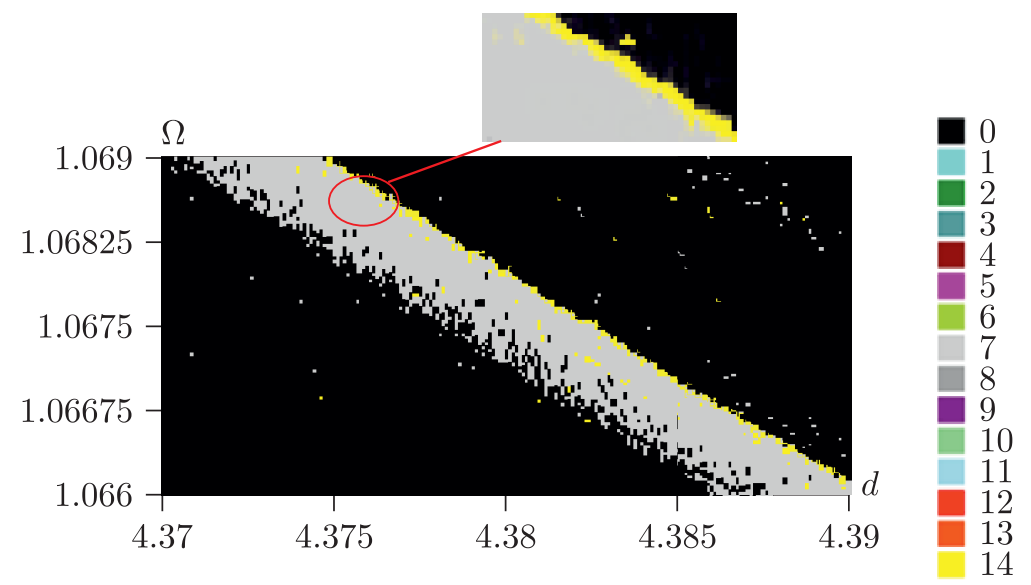

Fig. 6. The chart of dynamical regimes for the Poincaré map shown in Fig. 5.

For a more complete picture, Fig. 7 shows the appearance of a strange attractor for the values $\boldsymbol{K}=(-6,-3,-9), J=0, \delta=2, G=3$ at $d=4.385$ and $\Omega=1.06675 ; \Omega=1.0669$, $\Omega=1.07$ (the three-dimensional attractor is plotted in Fig. 5). 


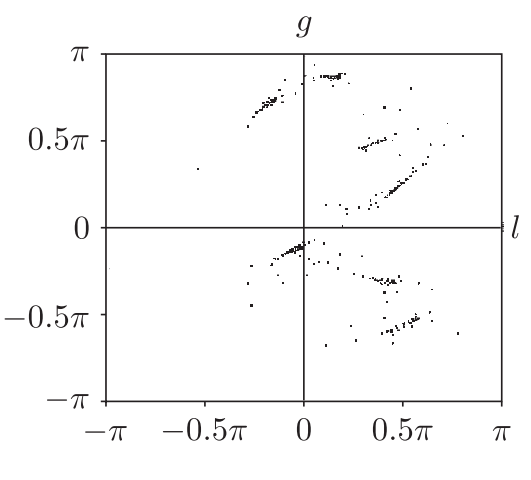

A

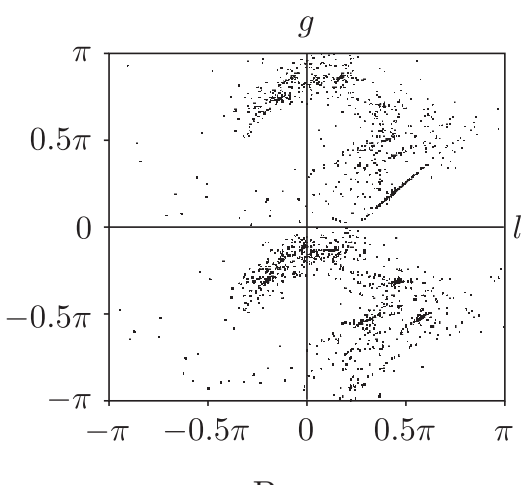

$\mathrm{B}$

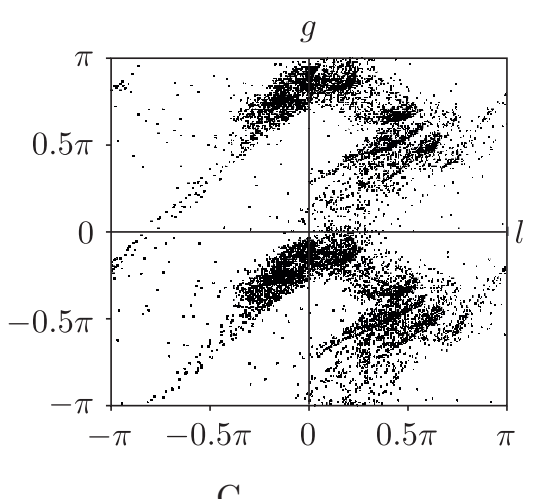

$\mathrm{C}$

Fig. 7. Two-dimensional projections of Poincaré maps for $\mathbf{I}=\operatorname{diag}(4,3,2), \boldsymbol{K}=(-6,-3,-9), d=4.385$, $J=0, \delta=2, G=3$ and for the A: $\Omega=1.06675, \mathrm{~B}: \Omega=1.0669$, C: $\Omega=1.07$.

\section{Conclusion}

The problem of motion without slippage of the Chaplygin ball with variable parameters has been considered. No strange attractors were found in numerical experiments when the ball was moving from rest. In general, the system may exhibit chaotic behavior. To illustrate this fact, strange attractors were constructed in the Poincaré map, and the trajectory of the contact point and Lyapunov exponents were determined. A chart of dynamical regimes was also constructed to determine the nature of the strange attractors. However, due to the large values of periods, only a doubling of the period 7-14 was detected. Therefore, the question of the Feigenbaum mechanism for the appearance of a strange attractor remains open.

The Poincaré maps have been constructed using the software package "Computer dynamics: Chaos" (see http://cd.ics.org.ru/chaos_pack/).

\section{References}

[1] Chaplygin, S. A., On a Ball's Rolling on a Horizontal Plane, Regul. Chaotic Dyn., 2002, vol. 7, no. 2, pp. 131-148; see also: Math. Sb., 1903, vol.24, no.1, pp.139-168.

[2] Moskvin, A. Yu., Chaplygin's Ball with a Gyrostat: Singular Solutions, Nelin. Dinam., 2009, vol. 5, no. 3, pp. 345-356 (Russian).

[3] Bolotin, S. V., The Problem of Optimal Control of a Chaplygin Ball by Internal Rotors, Regul. Chaotic Dyn., 2012, vol.17, no. 6, pp. 559-570.

[4] Bizyaev, I. A., Borisov, A. V., and Mamaev, I. S., Dynamics of the Chaplygin Ball on a Rotating Plane, Russ. J. Math. Phys., 2018, vol. 25, no. 4, pp. 423-433.

[5] Borisov, A.V., Kilin, A. A., and Mamaev, I. S., How to Control Chaplygin's Sphere Using Rotors: 2, Regul. Chaotic Dyn., 2013, vol.18, nos. 1-2, pp. 144-158.

[6] Moshchuk, N. K., On the Motion of Chaplygin's Sphere on a Horizontal Plane, J. Appl. Math. Mech., 1983, vol. 47, no. 6, pp. 733-737; see also: Prikl. Mat. Mekh., 1983, vol. 47, no. 6, pp.916-921.

[7] Mamaev, I. S. and Vetchanin, E. V., Dynamics of Rubber Chaplygin Sphere under Periodic Control, Regul. Chaotic Dyn., 2020, vol. 25, no. 2, pp. 215-236.

[8] Bizyaev, I. A., Borisov, A.V., and Mamaev, I. S., Different Models of Rolling for a Robot Ball on a Plane As a Generalization of the Chaplygin Ball Problem, Regul. Chaotic Dyn., 2019, vol. 24, no. 5 , pp. 560-582. 
[9] Borisov, A. V., Kilin, A. A., and Mamaev, I. S., The Problem of Drift and Recurrence for the Rolling Chaplygin Ball, Regul. Chaotic Dyn., 2013, vol. 18, no. 6, pp. 832-859.

[10] Karavaev, Yu. L. and Kilin, A. A., The Dynamics of a Spherical Robot of Combined Type by Periodic Control Actions, Russian J. Nonlinear Dyn., 2019, vol. 15, no. 4, pp. 497-504.

[11] Putkaradze, V. and Rogers, S., On the Optimal Control of a Rolling Ball Robot Actuated by Internal Point Masses, J. Dyn. Sys. Meas. Control, 2020, vol. 142, no. 5, 051002, 22 pp.

[12] Bai, Y., Svinin, M., and Yamamoto, M., Dynamics-Based Motion Planning for a Pendulum-Actuated Spherical Rolling Robot, Regul. Chaotic Dyn., 2018, vol. 23, no. 4, pp. 372-388.

[13] Kilin, A. A., Pivovarova, E. N., and Ivanova, T. B., Spherical Robot of Combined Type: Dynamics and Control, Regul. Chaotic Dyn., 2015, vol.20, no. 6, pp.716-728.

[14] Borisov, A. V., On the Liouville Problem, in Numerical Modelling in the Problems of Mechanics, Moscow: Mosk. Gos. Univ., 1991, pp. 110-118 (Russian).

[15] Vetchanin, E. V. and Mikishanina, E. A., Vibrational Stability of Periodic Solutions of the Liouville Equations, Russian J. Nonlinear Dyn., 2019, vol. 15, no. 3, pp. 351-363.

[16] Borisov, A. V., Kilin, A. A., and Pivovarova, E. N., Speedup of the Chaplygin Top by Means of Rotors, Dokl. Phys., 2019, vol.64, no. 3, pp. 120-124; see also: Dokl. Akad. Nauk, 2019, vol. 485, no. 3, pp. 285-289.

[17] Kozlov, V.V., The Euler-Jacobi-Lie Integrability Theorem, Nelin. Dinam., 2013, vol.9, no.2, pp. 229-245 (Russian).

[18] Markeev, A. P., Integrability of the Problem of Rolling of a Sphere with a Multiply Connected Cavity Filled with an Ideal Fluid, Izv. Akad. Nauk SSSR. Mekh. Tverd. Tela, 1986, vol.21, no. 1, pp. 64-65 (Russian).

[19] Borisov, A. V. and Mamaev, I.S., Rigid Body Dynamics, De Gruyter Stud. Math. Phys., vol.52, Berlin: De Gruyter, 2018.

[20] Feigenbaum, M. J., Quantitative Universality for a Class of Nonlinear Transformations, J. Statist. Phys., 1978, vol. 19, no. 1, pp. 25-52. 